\title{
Uso da dieta de eliminação no tratamento de Esofagite Eosinofílica: uma revisão de
}

\section{literatura}

\author{
Use of elimination diet in the treatment of Eosinophilic Esophagitis: a review of the literature
}

Uso de dieta de eliminación en el tratamiento de la Esofagitis Eosinofílica: una revisión de la

literatura

Recebido: 08/10/2021 | Revisado: 13/10/2021 | Aceito: 20/10/2021 | Publicado: 22/10/2021

\author{
Kelly Vanderlei Macedo \\ ORCID: https://orcid.org/0000-0001-8729-1282 \\ Centro Universitário Santo Agostinho, Brasil \\ E-mail: kvanderleimacedo@gmail.com \\ Daniele Rodrigues Carvalho Caldas \\ ORCID: https://orcid.org/0000-0001- 8830-0283 \\ Universidade Federal do Piauí, Brasil \\ E-mail: danielerccaldastrabalho@gmail.com \\ Liejy Agnes dos Santos Raposo Landim \\ ORCID: https://orcid.org/0000-0002-8214-2832 \\ Centro Universitário Santo Agostinho, Brasil \\ E-mail: liejylandim@gmail.com
}

\begin{abstract}
Resumo
A Esofagite Eosinofílica (EEo) é uma patologia que vem crescendo ao longo do tempo. Epidemiologicamente ocorre de forma variada tanto geograficamente quando por faixas etárias, com incidência maior em adultos que em crianças. Os sintomas são baseados no quadro diagnóstico combinado com algumas características clínicas, podendo ser confundidos com a doença do refluxo gastroesofágico (DGRE). O presente estudo trata-se de uma revisão integrativa sobre as modificações das dietas aplicadas em pacientes com EEo. Foram selecionados artigos indexados dos últimos 10 anos, disponibilizados na íntegra em idiomas inglês, português e espanhol. A coleta de dados ocorreu nas bases: Scientific Electronic Library Online (SciELO), Biblioteca Virtual de Saúde (BVS), National Library of Medicine (PubMED), Medical Literature Analysis and Retrieval System Online (Medline) e Google Acadêmico. Após a aplicação dos critérios de exclusão e inclusão, 6 artigos formam selecionados para análise e discussão. A partir dos estudos, concluiu-se que houve a melhora do quadro clínico dos pacientes estudados a partir da adesão das dietas de exclusão e dietas de eliminação empíricas. Alimentos como o ovo, trigo, glúten e o leite foram os que mais influenciavam na EEo, consequentemente influenciando nos sintomas. Portanto, o acompanhamento e adesão de forma contínua da dieta recomendada ao paciente com EEo é de suma importância para seu tratamento e resultado da redução de sua sintomatologia.
\end{abstract}

Palavras-chave: Esofagite eosinofílica; Refluxo Gastroesofágico; Dietas.

\begin{abstract}
Eosinophilic Esophagitis (EEo) is a pathology that has been growing over time. Epidemiologically it occurs variably both geographically and by age groups, with a higher incidence in adults than in children. The symptoms are based on the diagnostic picture combined with some clinical features, and can be confused with gastroesophageal reflux disease (GERD). The present study is an integrative review on dietary modifications in patients with EEo. We selected indexed articles from the last 10 years, available in full in English, Portuguese and Spanish. Data collection occurred in the following databases: Scientific Electronic Library Online (SciELO), Virtual Health Library (VHL), National Library of Medicine (PubMED), Medical Literature Analysis and Retrieval System Online (Medline) and Google Scholar. After applying the exclusion and inclusion criteria, 6 articles were selected for analysis and discussion. From the studies, it was concluded that there was improvement in the clinical picture of the patients studied from the adherence to exclusion diets and empirical elimination diets. Foods such as egg, wheat, gluten and milk were the most influential in the EEo, consequently influencing the symptoms. Therefore, the continuous monitoring and adherence to the recommended diet for patients with EEo is of utmost importance for their treatment and the result of reducing their symptoms.
\end{abstract}

Keywords: Eosinophilia esophagitis; Gastro esophageal Reflux; Diets. 


\begin{abstract}
Resumen
La esofagitis eosinofílica (EEo) es una patología que ha ido creciendo con el tiempo. Epidemiológicamente se presenta de forma variada tanto geográficamente como por grupos de edad, con una mayor incidencia en adultos que en niños. Los síntomas se basan en el cuadro diagnóstico combinado con algunas características clínicas, y pueden confundirse con la enfermedad por reflujo gastroesofágico (ERGE). El presente estudio es una revisión integradora sobre las modificaciones de las dietas aplicadas en pacientes con EEo. Se trata de una selección de artículos indexados de los últimos 10 años, disponibles en su totalidad en inglés, portugués y español. La recogida de datos se realizó en las bases de datos: Scientific Electronic Library Online (SciELO), Virtual Health Library (BVS), National Library of Medicine (PubMED), Medical Literature Analysis and Retrieval System Online (Medline) y Google Scholar. Tras aplicar los criterios de exclusión e inclusión, se seleccionaron 6 artículos para su análisis y discusión. A partir de los estudios, se concluyó que hubo una mejora en el cuadro clínico de los pacientes estudiados a partir de la adherencia a las dietas de exclusión y a las dietas de eliminación empíricas. Alimentos como el huevo, el trigo, el gluten y la leche fueron los que más influyeron en la EEo, influyendo consecuentemente en los síntomas. Por lo tanto, el seguimiento continuo y el cumplimiento de la dieta recomendada para los pacientes con EEo es de suma importancia para su tratamiento y el resultado de la reducción de sus síntomas.
\end{abstract}

Palabras clave: Esofagitis eosinofílica; Reflujo gastroesofágico; Dietas.

\title{
1. Introdução
}

A esofagite eosinofílica (EoE) é definida como uma patologia crônica do esôfago, caracterizada por um processo inflamatório da parede do esôfago. Foi descrita pela primeira vez em 1970, e se manifesta clinicamente por uma disfunção esofagiana e histologicamente, por uma inflamação predominantemente eosinofílica (Lucendo, et al., 2017). É notório que ao longo do tempo vem crescendo os estudos sobre os casos clínicos e diretrizes a respeito da abordagem, fisiopatologia e forma de tratamento vem se destacando (Pesek \& Gupta, 2018).

Outra vertente, conforme traz Mendonça et al. (2020), indica que a EoE resulta de um processo inflamatório, imunomediado, em resposta a alimentos ou aeroalergénios específicos, não existindo ainda um método ideal que permita identificar quais são os alimentos que estão envolvidos nesta patologia. A esofagite eosinofílica é vista, por autores como (Liacouras, 2011; Pulgar, 2016; Teixeira, 2020), como doença crônica com antígeno mediado a sensibilização a alimentos, que causam sintomas decorrentes da disfunção esofágica causando inflamação.

A fisiopatologia desta doença ainda necessita de mais estudos, pois não se sabe ao certo como ela se apresenta existindo lacunas a serem respondidas. Porém, existem estudos que dizem que a EEo está associada a fatores ambientais e a predisposição genética. Essas possíveis alterações genéticas estão associadas ao polimorfismo de um único nucleotídeo no gene CCL2617 ou simplesmente o gene que codifica a eotaxina-3, sendo ela uma molécula efetora para a EEo sendo patogênese da doença, onde é altamente expressiva em células epiteliais esofágicas de pacientes afetados (Veiga, et al., 2017).

Existem citocinas que estão associadas a inflamação da EEo, em especial a linfopoetina estromal tímica (TSLP) que é um regulador fundamental da inflamação alérgica do tipo Th-2. A mesma, é secretada por células de linhagem não hematopoiética, células essas epiteliais, fibroblastos e células de músculo liso em resposta a citocinas atópicas (IL-4, IL13, TNFa) e alérgenos ambientais. Podendo estar associado a doenças alérgicas, variando de indivíduo para indivíduo ou mesmo acredita-se que eles possam estar associados a edema, inflamação e possível fibrose do esôfago (Barros, et al., 2018; Molina, et al., 2011).

Além da esofagite eosinofílica, existem mais 3 tipos de esofagite. A Esofagite de refluxo ou esofagite erosiva, que ocorre quando a válvula do aparelho digestivo não funciona de forma correta, essa válvula existente no aparelho digestivo não consegue evitar que os ácidos existentes no estômago voltem para o esôfago, causando erosões/feriadas com o tempo (Almeida, 2019). Esse tipo de esofagite pode ocorrer por agentes de forma extrínseca ou intrínseca, sendo associado a DRGE (Coelho, 2020). Sendo causadas também por outras alterações associada a dificuldade da limpeza esofágica ou a não regressão contrátil esofágica (Almeida, 2019). 
A Esofagite por medicamento, por sua vez é causada pelo alto uso de medicamentos que causam danos no tecido do esôfago. A grande variedade de tranquilizantes, sedativos e anestésicos, por exemplo, tem a capacidade de diminuir o tônus do esfíncter esofágico inferior (EEI), sendo o mecanismo de defesa natural do organismo e responsável por impedir o refluxo de conteúdo gástrico para o esôfago (Rodríguez-Alarcón, et al., 2015).

Hoversten, et al. (2018) informa que a esofagite infecciosa vem a ser a terceira principal causa de esofagite, após a DRGE e a esofagite eosinofílica. A mesma se manifesta mais em paciente com sistema imunológico baixo, ou seja, imunocomprometido, no entanto também podem atingir pessoas imunocompetentes. Sendo paciente do sexo masculino os mais afetados; esse tipo de esofágia se manifesta por odinofagia e disfagia, com sintomas de náuseas, vômitos e dor retroesternal, sendo em sua grande maioria causada por cândida, vírus citomegálico (VCM), vírus herpes e o próprio refluxo (Cezar, et al., 2016).

Tratando-se epidemiologicamente da EEo sua prevalência ocorre de forma variada tanto geograficamente quanto em grupos de diversas faixas etárias, tendo alta incidência em adultos do que em relação a crianças (Arias, et al., 2016). A causa mais frequente da esofagite está associada a esofagite crônica, logo depois à doença de refluxo gastroesofágico (DRGE), sendo a primeira causa de disfagia e impactação alimentares acometidas em crianças, adolescentes e jovens adultos (Lucendo, et al., 2017).

Seus sintomas são similares a doença do refluxo gastroesofágico (DRGE), com infiltração eosinofílica do epitélio que vem a prejudicar a integridade da barreira epitelial esofágica. Mesmo assim, estudiosos da área procuram verificar e identificar preditores independentes de EEo em distinção à DRGE, que incluem: idade, se é mais jovem ou não; atopia; alergia e características endoscópicas, tais como anéis, sulcos, placas e exsudato (Rawla, et al., 2018).

Pereira, et al. (2019) alega que a EEo provoca aumento da sensibilidade do esôfago ao ácido e a exposição à alergênicos locais, não existindo comprometimento de outros segmentos do trato gastrointestinal. O diagnóstico da EEo inclui diversos sintomas: disfagia, vômitos, dor abdominal e recusa alimentar, com ou sem desaceleração do crescimento esofágico; se referindo a parte clínica, o elevado número de eosinófilos deve ser observado, pois pode causar abscessos eosinófilos, criptite, fibrose. As biópsias da mucosa esofágica são realizadas a fim de averiguar a quantidade de eosinófilos, com pelo menos 15 eosinófilos/campo onde vem a indicar um grande aumento e certa persistência da eosinofílica, em seguida o tratamento é realizado com altas doses de inibidores de bombas de prótons (Groetch, et al., 2017).

As atuais formas de tratamento da EEo se baseiam na terapia dietética, com o uso de dietas de eliminação baseadas em testes de alergia, para a eliminação de 6 alimentos (leite, glúten, ovo, peixes, frutos- do- mar e frutos secos e trigo), a dieta de eliminação guiada e a dieta elementar. A Dieta elementar consiste na eliminação por completa de todos os alimentos que possam causar alergia alimentar e é baseada em aminoácidos por um período de seis semanas; enquanto a dieta de restrição empírica consiste na eliminação de alimentos frequente na patologia EEo, como leite de vaca, soja, ovo, trigo, amendoim, castanha, peixes, frutos do mar sendo ela isolado ou combinada (Ferreira, et al., 2019).

Alimentos como o trigo, leite, ovo e glúten a exemplos apresentam-se como fatores associados a alergia alimentar em decorrência de fatores associados a anticorpos IgEs específicos para eles ou por reações imediatas. O trigo, a exemplo, em decorrência das diversas proteínas existentes em sua composição pode desencadear a alergia alimentar, uma vez que essas proteínas são resistentes em altas temperaturas (Cianferoni, 2016).

A intolerância ao leite está dividida em 3 fases (congênita que é relacionada a alteração genética, primária aquela que ocorre devido a modificações na genética do indivíduo e secundária que ocorre em decorrência de alguma patologia gastrointestinal, que provocam má absorção da lactose provocando no intestino grosso um aumento da pressão osmótica que causa a saída dos eletrólitos e água, causando diarreias (Rienzo, et al., 2013). 
É importante destacar que quando se trata de alimentos como o leite e glúten fala-se de intolerância alimentar, que se distingue de alergia alimentar no que se refere aos agravamentos dos sintomas. No entanto, também pode-se falar em alergia a proteína do leite (APVL) que atinge ao sistema imunológico (Monteiro, et al., 2019). Por sua vez, a intolerância ao glúten está relacionado a própria alergia ao trigo, intolerância ao glúten não-celíaca, patologias ou mesmo ao uso exacerbado de pesticidas provocando assim uma doença autoimune relacionado aos genes HLA-DQ2 e DQ8 (Tolvoli, et al., 2015).

Kotchetkoff, et al. (2020) traz em recente estudo que um dos fatores a ser levado em consideração na alergia ao ovo e ao leite é a tolerância a esses alimentos em sua forma termicamente tratada, relatando ainda que indivíduos com o fenótipo AA mediado por IgE podem ser tolerantes ao leite de vaca e ao ovo quando descoberto essas tolerâncias na infância.

Com isso, este trabalho objetiva analisar a melhor forma de tratamento não farmacológico (dieta) para pacientes que tenham esofagite eosinofílica e se o tratamento baseado em uma dieta com restrição de leite, glúten, soja e trigo, auxilia na melhora da sintomatologia da doença.

\section{Metodologia}

Este estudo trata-se de uma revisão integrativa da literatura com abordagem teórica. Trata-se de uma pesquisa que aborda a dietoterapia no tratamento de pacientes adultos com EEo. Pereira et al. (2018) informa que o estudo de caso trata-se de uma metodologia que visa observar um fenômeno de forma individual ou coletiva por meio de processos sociais; o estudo pode ser quantitativo ou qualitativo onde seu objetivo é mostrar um melhor entendimento sobre o fenômeno que está sendo estudado.

Foi realizada uma busca das publicações, dos últimos 10 anos (entre 2011 a 2021), objetivando analisar sobre as dietas aplicadas no tratamento da esofagite eosinofílica em pacientes adultos nos bancos de dados da: Biblioteca Virtual de Saúde (BVS), Scientific Electronic Library Online (Scielo), Literatura Latino Americana e do Caribe em Ciências da Saúde (Lilacs), National Library of Medicine (PubMED) e Google Acadêmico. Foram usados os seguintes descritores: "esofagite eosinofílica", "alergia alimentar", "dieta", "dietoterapia", "alergia alimentar" e "doença do refluxo gastroesofágico", os idiomas usados foram português, inglês e espanhol adaptados de acordo com a base de dados usada. Foram três etapas ao todo no desenvolvimento do estudo: a primeira iniciou com a busca do descritor "esofagite eosinofílica", logo após o descritor "alergia alimentar", "doença do refluxo gastroesofágico" e "dieta" todos utilizando-se de operador booleano AND. Foram excluídas monografias, artigos não relacionados a temática, revisões bibliográficas e livros.

No banco de dados BVS quando usado o descritor de dados "esofatis eosinofílica" encontrou-se de forma em geral 1691 textos completos, sendo 1593 na base de MEDLINE, 57 na base de dados IBECS, 39 na LILAOS, 2 LIPECS, 1 BRAVANCS, 1 RED TESA e 1 no Sec. De Saúde de SP. Logo após, filtrou-se ainda mais o campo com o seguinte descritor "dietoterapia" onde foram encontrados 31 no total, nas bases de dados MEDLINE foram 30 e IBECS 1, sendo 29 escritos em inglês e 2 alemães. Foram excluídos artigos que não abordassem a temática de esofagite eosinofílica em adultos, restando apenas 1 que foi utilizado.

Nos demais bancos de dados Scielo, PubMed, Lilasc e Google acadêmico foram encontrados 30 artigos que abordavam os descritores, porém foram excluídos 23 artigos de acordo com os critérios de exclusão e inclusão. Resultando então em 6 artigos para compor esta revisão (Figura 1). 
Figura 1 - Fluxograma do processo de busca e seleção dos estudos inclusos na revisão Teresina / PI, Brasil, 2021.

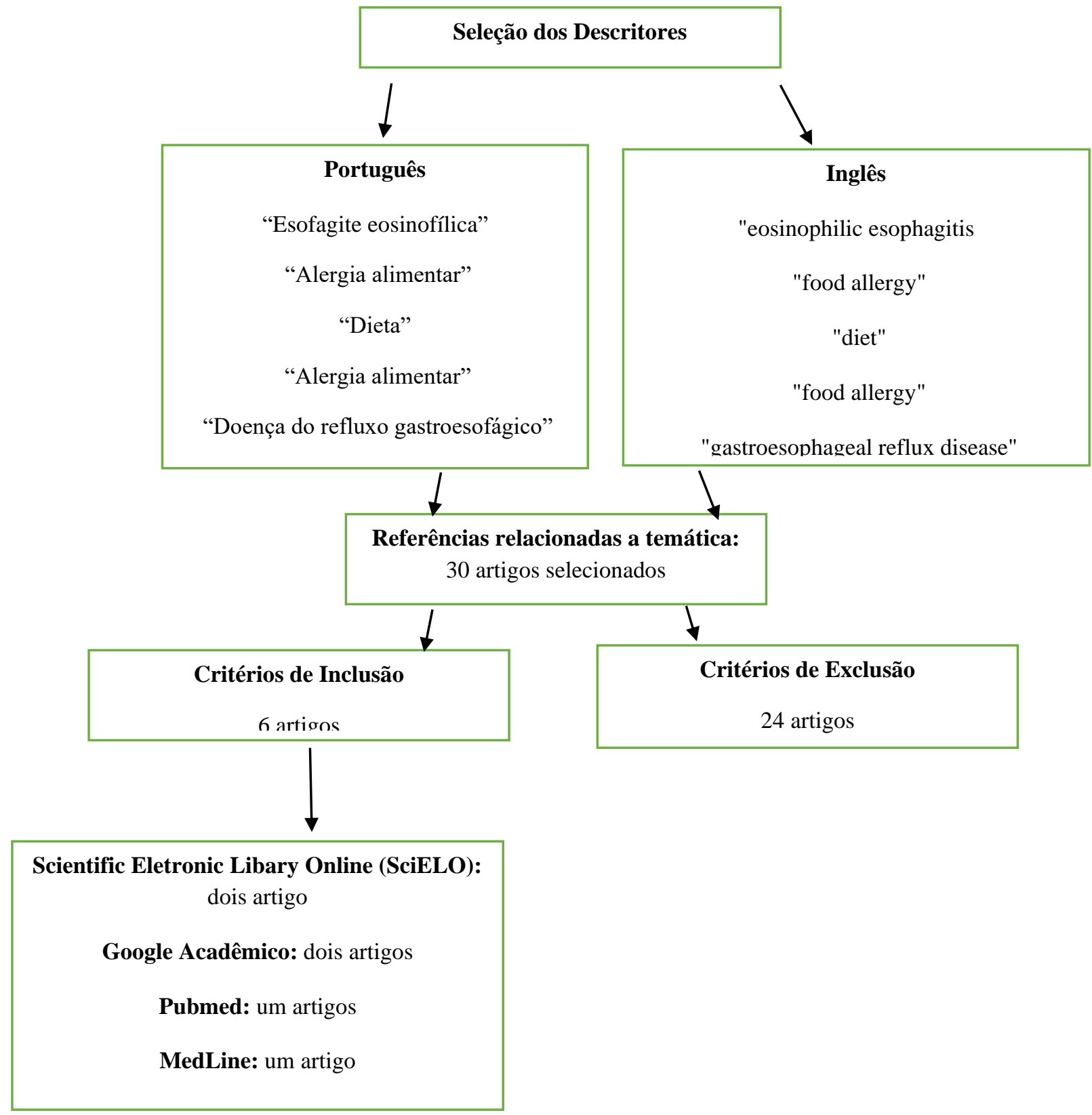

Fonte: Dados da Pesquisa (2021).

\section{Resultados e Discussão}

Em todos os estudos analisados é possível constatar a relação dos autores quanto ao uso de dietas de exclusão e eliminação no tratamento dietético de pacientes com a esofagite eosinofílica (EEo). Além disso, foi possível observar que alimentos como leite, o ovo, trigo, glúten foram os percussores da EEo nos pacientes que se submeteram aos estudos, com resultados satisfatórios em pacientes que obedeciam a deita de exclusão de 4 a 6 alimentos. No entanto, quando essa dieta não era seguida conforme indicado, a remissão dos eosinófilos não regredia. Vale ressaltar que, caso essa temática seja trabalhada a longo prazo, resultados mais precisos podem surgir.

Na Tabela 1 estão expostos os principais resultados sobre as dietas aplicadas a pacientes adultos com esofagite eosinofílica. 
Tabela 1 - Produções cientificas publicadas de 2012 a 2021 segundo autor, título, objetivo, tipo de estudo e resultados.

\begin{tabular}{|c|c|c|c|c|}
\hline Autor / ano & Título & Objetivos & $\begin{array}{l}\text { Tipo de } \\
\text { Estudo }\end{array}$ & Resultados \\
\hline $\begin{array}{l}\text { Molina-Infante, et } \\
\text { al. (2012) }\end{array}$ & $\begin{array}{l}\text { A dieta de eliminação } \\
\text { seletiva com base em testes } \\
\text { cutâneos tem eficácia abaixo } \\
\text { do ideal para esofagite } \\
\text { eosinofílica adulta. }\end{array}$ & $\begin{array}{l}\text { Analisar a aplicação de } \\
\text { dietas nos pacientes com } \\
\text { EEo. }\end{array}$ & Prospectivo & $\begin{array}{l}\text { Dieta a dieta de eliminação de } 6 \text { alimentos com } \\
\text { reintrodução de alimentos caminha para resultados } \\
\text { favoráveis para adultos com EEo, a de eliminação com } \\
\text { testes cutâneos mostrou-se pouco positivos. }\end{array}$ \\
\hline $\begin{array}{c}\text { Gonsalves, et al. } \\
\text { (2012) }\end{array}$ & 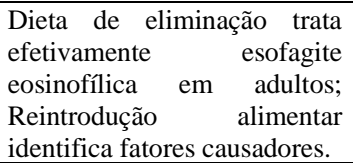 & $\begin{array}{l}\text { Avaliar a repostas } \\
\text { histológica da dieta de } \\
\text { seis alimentos (SFED) } \\
\text { que apresentam EEo em } \\
\text { adultos. }\end{array}$ & Prospectivo & $\begin{array}{l}\text { Com uma dieta de eliminação nos pacientes houve } \\
\text { melhora e redução nos sintomas da EEo nos pacientes } \\
\text { avaliados. }\end{array}$ \\
\hline $\begin{array}{l}\text { Lucendo, et al. } \\
\qquad(2013)\end{array}$ & $\begin{array}{l}\text { Dieta de eliminação empírica } \\
\text { de } 6 \text { alimentos induzida e } \\
\text { mantida remissão prolongada } \\
\text { em pacientes com esofagite } \\
\text { eosinofílica adulta: um estudo } \\
\text { prospectivo sobre a causa } \\
\text { alimentar da doença. }\end{array}$ & $\begin{array}{l}\text { Avaliar a eficácia de } 6 \\
\text { dieta de eliminação de } \\
\text { alimentos na indução e } \\
\text { manutenção da remissão } \\
\text { prolongadas } \quad \text { em } \\
\text { pacientes com EoE } \\
\text { adulto. }\end{array}$ & $\begin{array}{c}\text { Descritivo e } \\
\text { experimental. }\end{array}$ & $\begin{array}{l}\text { Encontrou-se um único antígeno alimentar ofensivo } \\
\text { identificado em } 35,71 \% \text { dos pacientes, } 2 \text { gatilhos } \\
\text { alimentares foram identificados em } 30,95 \% \text { e } 3 \text { ou } \\
\text { mais gatilhos alimentares foram identificados em } \\
33,3 \% \text {. O leite de vaca foi o antígeno alimentar mais } \\
\text { comum }(61,9 \%) \text {, seguido pelo trigo }(28,6 \%) \text {, ovos } \\
(26,2 \%) \text { e leguminosas }(23,8 \%) \text {. }\end{array}$ \\
\hline $\begin{array}{l}\text { Higuera-Pulgar, et } \\
\text { al. (2016) }\end{array}$ & $\begin{array}{l}\text { Ingesta dietética de pacientes } \\
\text { adultos con esofagitis } \\
\text { eosinofílica que siguen una } \\
\text { dieta de exclusión de seis } \\
\text { grupos de alimentos. }\end{array}$ & 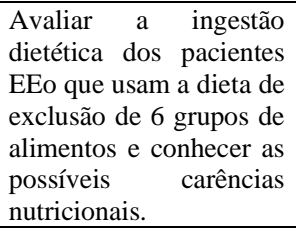 & $\begin{array}{l}\text { Estudo } \\
\text { transversal } \\
\text { Descritivo }\end{array}$ & $\begin{array}{l}\text { Observou-se } 14 \text { pacientes, onde forma submetidos a } \\
\text { dieta de exclusão de } 6 \text { grupos de alimentos. Verificou- } \\
\text { se que a dieta de exclusão proporcionou deficiência } \\
\text { em relação a energia, proteínas, fibra, micronutrientes } \\
\text { (cálcio, zinco, magnésio, ácido fólico, niacina, } \\
\text { vitaminas B2 e D). }\end{array}$ \\
\hline Reed, et al. (2017) & $\begin{array}{l}\text { As dietas de eliminação de } \\
\text { alimentos são eficazes para o } \\
\text { tratamento de longo prazo de } \\
\text { adultos com esofagite } \\
\text { eosinofílica }\end{array}$ & $\begin{array}{l}\text { Avaliar os resultados a } \\
\text { longo prazo das dietas } \\
\text { de eliminação de } \\
\text { alimentos para o } \\
\text { tratamento de adultos } \\
\text { coma EEo. }\end{array}$ & $\begin{array}{l}\text { Estudo de } \\
\text { coorte } \\
\text { retrospectivo. }\end{array}$ & $\begin{array}{l}\text { Avalizou-se } 52 \text { pacientes onde foram feitos a } \\
\text { distribuição de dietas de eliminação por grupos a elas. } \\
\text { A dieta elementar atingiu resultado de remissão } \\
\text { histológica em } 91 \% \text {, a dieta de eliminação empírica } \\
\text { em } 72 \% \text { e as dietas direcionadas a teste de alergia } \\
46 \% \text {. Mostrando que a aplicação da dieta elementar, } \\
\text { assim como a dietoterapia e manutenção da merma } \\
\text { reduz os sintomas histologicamente a um longo prazo. }\end{array}$ \\
\hline $\begin{array}{l}\text { Molina-Infante, et } \\
\text { al. (2017) }\end{array}$ & $\begin{array}{l}\text { Dieta de eliminação empírica } \\
\text { intensificada para esofagite } \\
\text { eosinofílica pediátrica e } \\
\text { adulta: } O \text { estudo } 2-4-6 .\end{array}$ & $\begin{array}{l}\text { Avaliar a eficácia de } \\
\text { uma estratégia alimentar } \\
\text { intensificada para o } \\
\text { EoE. }\end{array}$ & $\begin{array}{c}\text { Estudo } \\
\text { prospectivo }\end{array}$ & $\begin{array}{l}\text { Observou-se que } 97 \text { dos pacientes avaliados } \\
\text { completaram todas as fases do estudo da dieta dos } \\
\text { grupos de } 2 \text { alimentos (TFGED) onde alcançaram } \\
\text { remissão do EoE em } 56(43 \%) \text { pacientes, sem } \\
\text { diferenças entre as idades. Os gatilhos alimentares nos } \\
\text { respondentes do TFGED foram leite }(52 \%) \text {, grãos que } \\
\text { contêm glúten (16\%) e ambos }(28 \%) \text {. EoE induzido } \\
\text { apenas pelo leite esteve presente em } 18 \% \text { e } 33 \% \text { dos } \\
\text { adultos e crianças, respectivamente. As taxas de } \\
\text { remissão com a dieta de eliminação do grupo de } 4 \\
\text { alimentos (FFGEDs) e grupos de } 6 \text { alimentos da dieta } \\
\text { de eliminação (SFGEDs) foram de } 60 \% \text { e } 79 \text {. }\end{array}$ \\
\hline
\end{tabular}

Fonte: Dados da pesquisa (2021).

Molina-Infante et al. (2012) realizaram um estudo prospectivo em 22 adultos com EoE, onde abordaram a eficácia da dieta de eliminação, nesse estudo os pacientes foram submetidos a teste de puntura, patch test e prick to prick e a atopia foram definidos tanto neles quando em suas famílias. Usou-se 26 alimentos diferentes, tais como: leite, ovo, grãos (trigo, milho, centeio, cevada, aveia e arroz), amendoins, leguminosas (soja, lentilhas, ervilhas, e feijão), carnes (carne de vaca, frango e porco), legumes (batata e tomate), marisco (camarão), peixe (pescada), e frutas (melão, kiwi, maçã, morango, banana, e pêssego), os pacientes que relutaram em realizar a dieta tiveram resultados negativos ou não vieram a responder a dieta de eliminação, por outro lado aqueles que seguiram a dieta de eliminação baseada em teste cutâneo tiveram eficácia.

Molina- Infante et al. (2012) descreveram que através da dieta de eliminação somente 4 pacientes responderam a dieta. Essa baixa resposta a dieta de eliminação de 6 alimentos com reintrodução deve-se a alguns fatores: por serem longas e caras, além de ter várias inserções de endoscopias superiores possa ter contribuído para a desistência dos demais participantes da pesquisa. Outro fator foi o tempo de protocolo adotado de 6 semanas que foram verificados. Isso pode estar associado a sensibilização da dieta de eliminação seletiva por teste cutâneo dos aeroalergénios em adultos. O uso dessa dieta ainda é 
questionável, nos mostrando que a terapia voltada somente para esse tipo de dieta aplicada em adultos ainda precisa de mais estudos.

Gonsalves et al. (2012) trazem outro estudo que corrobora com a tese dos autores acima, por meio da avaliação e observação da dieta de eliminação de 6 alimentos onde os pacientes evitaram a ingestão de leite, soja, ovo, trigo, amendoim, nozes, mariscos e peixes aplicado aos 50 adultos que participaram do estudo, também por um período de 6 semanas os participantes foram submetidos também a esofágicos, teste de picada de alimentos, biopsias e aeroalergénios para acompanhar a evolução da remissão dos sintomas da EEo. Somente 20 concluíram o estudo com a reintrodução dos alimentos que haviam sido eliminados, todos antes da aplicação da dieta de eliminação passaram pelo teste de picada (SPT) de alimentos e aero alergênicos.

Gonsalves et al. (2012) obtiveram alto grau de eficácia do SFED no tratamento de ambos os sintomas e histopatológica em adultos com EoE. Vinte dos pacientes que se submeteram aos testes (70\%) deles alcançaram mais de 50\% de redução no pico de eosinófilo, $70 \%$ alcançando 10 eos/hpf e $64 \%$ alcançando 5 eos/hpf após o tratamento.

Lucendo et al. (2013) reforça que pacientes que seguiram a dieta de exclusão tiveram uma significativa eficácia associada a dieta de eliminação de 6 alimentos, descrito em seu trabalho com a sigla (SFED) da contagem de eosinófilos. Dos 67 pacientes tratados, 37 alcançaram uma histologia de densidade eosinofílica menor de 15 eosinófilos- hpf e em 12 pacientes exibiram uma densidade de eosinófilos de 7,78 +2,26. Os 18 pacientes restantes não demonstraram diferenças significativas na contagem média de eosinófilos esofágicos antes da dieta.

Nesse processo da pesquisa Luceno et al. (2013) informam que por meio da reintrodução alimentar, observou-se dos 49 pacientes que seguiram esse sequencial havendo um único antígeno alimentar ofensivo; em 15 pacientes, 2 alimentos agressivos. Nenhum dos pacientes completaram o desafio de exclusão dos 6 alimentos (leite, trigo, ovos e leguminosas). Esses mesmos alimentos foram considerados como gatilhos mais identificados nos pacientes. Apesar do resultado descrito, o estudo de Luceno corrobora com o estudo de Molina-Infante et al. (2012) em relação a remição de eosinófilos associado a dieta de exclusão.

Fernandes et al. (2018) informa que a dieta de invictação baseada em TCP ou dosagem de IgE específicos induz a remissão histológica em menos de 1/3 nos adultos e um pouco superior em crianças. Dependendo do alimento avaliado o resultado é positivo de $26,3 \%$ a $86,3 \%$. Sendo negativo com $90 \%$ para alimentos como o ovo, trigo, soja e o leite.

No caso analisado, os autores concluem que em relação ao diagnóstico de défice de IgA foi um achado incidental e que o doente não apresenta qualquer sintoma relacionado a patologia, sendo assim, não necessitando de uma terapia especifica, somente conscientização e educação sobre as potenciais reações anti-inflamatórias que possam surgir. Uma vez que histopatologicamente a EEo existe em geral, um infiltrado inflamatório crônico que vem a incluir eosinófilos, mastócitos, basófilos e células T, a produzirem citocinas do perfil Th2 (IL-4 e IL-13) que promovem inflamação e disfunção adiciona (Dellon et al., 2018).

Nos estudos que avaliaram a dieta de restrição, para corroborar com os dados de (Fernandes, 2018), de acordo com resultados de testes alérgicos (teste de puntura e patch test) Simon et al., em 2006, descreveu o resultado do estudo feito com seis adultos, todos com EEo, que estavam sensibilizados à gramínea, centeio e trigo. Os indivíduos evitaram centeio e trigo por 6 semanas, e apenas 1 paciente apresentou melhora em seus sintomas clínicos, em adultos o teste foi mais limitado (Veiga et al., 2017).

Higuera-Pulgar et al. (2016) em seu estudo demonstram que as tradicionais dietas de exclusão favorecem a remissão histológica dos eosinófilos, assim como a ingestão inadequada da dieta de exclusão de 6 alimentos (leite, ovos, peixes, marisco, legumes, frutos secos e cereais) que podem causar deficiências nutricionais nos pacientes que são submetidos a essas 
restrições. Nesse sentido necessita-se um estudo aprofundado sobre as deficiências nutricionais existentes na dieta de exclusão para verificar as demais consequências que podem acometer no indivíduo.

Reed et al. (2017) por sua vez, avaliaram 52 pacientes, dentre elas mulheres brancas que foram submetidas a dieta empírica de eliminação de 6 alimentos e a testes de pele para verificação dos alimentos que lhes causavam gatilhos para os aparecimentos dos sintomas de EEo. A dieta de eliminação consistia em eliminar laticínios, ovos, trigo, soja, nozes e frutos do mar. Essa dieta foi aplicada por 6 semanas acompanhada por médicos e nutricionistas, o monitoramento era realizado por meio de endoscopia que averiguava o prognostico histológico.

Reed et al. (2017) avaliaram que nesse estudo que alguns alimentos da dieta elementar serviam como gatilhos para a EEo, tais como: leite, trigo, ovos. Nove pacientes em especifico passaram pelo teste de alergia onde foram encontrado antígenos alimentares nos alimentos (ovo, trigo, laticínios, frutos do mar, nozes, frango e em algumas frutos que os autores não descreveram. Os únicos alimentos que não foram considerados como gatilhos estão relacionados a carne de porco, aveia e carne bovina. Com isso os pesquisadores obtiveram uma melhora nos sintomas da EEo de $91 \%$ com a aplicação da dieta elementar, $72 \%$ com a dieta de eliminação empírica e as dietas relacionadas a teste de alergia $46 \%$; sendo que em $67 \%$ das pacientes ocorreu a redução de sintomas como disfagia, azia e dor no peito.

Molina-Infante et al. (2017) em seus estudos mostra que o uso de dietas de eliminação empíricas aplicado em distintos grupos se mostrou positivo. O autor fez sua pesquisa em 3 etapas: dieta de eliminação do grupo de 4 alimentos (FFGED) eliminando leite de vaca e trigo por seis semanas. Verificou-se então que houve uma remissão de $40 \%$ nos pacientes avaliados, após a reintrodução do leite, ovos e leguminosas, tanto em adultos quanto em crianças, sendo permitida ingestão de ovos e leguminosas. O leite foi o alimento que desenvolveu a doença em metade dos respondentes do TFGED.

Molina-Infante et al. (2017) também informa que logo após teve a intervenção intensificada FFGED obteve uma remissão nos pacientes avaliados de 60\%. Tanto no FFGED quanto SFGEDs foram encontrados de 1 a 2 gatilhos alimentares após a reintrodução dos alimentos restritos. Em relação ao SFGEDs houve 79\% de remissão baseadas nas dietas restritivas, no entanto a progressão dessa aplicação de dietas restritas deve ser progressiva. O leite apresentou-se como maior gatilho no EEo, foi o que mais apresentou nos testes, logo em seguia os grãos que contém glúten e o ovo. As leguminosas foram as que menos apareceram relevante a EEo.

\section{Considerações Finais}

Apesar da variedade de dietas existentes como forma de tratamento não farmacológico no tratamento de EEo, as dietas de exclusão assim como as dietas de eliminação empíricas foram as que mais apresentaram resultados nos estudos descritos acompanhados com as dietas guiadas por teste alergênico seguindo protocolo de 6 semanas, tendo respostas significativas nos pacientes que as seguiam.

Os estudos mostram que alimentos como o ovo, trigo, glúten e o leite foram os que mais influenciavam na EEo; consequentemente influenciando nos sintomas, o que confirma que a EEo está associada ao défice seletivo de IgA e doença alérgica, conforme relado pelos autores. $\mathrm{O}$ uso de bloqueadores de ácido associados a dieta de exclusão melhora progressivamente a sintomatologia dos pacientes com EEo, sendo adultos ou crianças. Portanto, o acompanhamento e adesão de forma contínua da dieta recomendada ao paciente com EEo é de suma importância para seu tratamento e resultado da redução de sua sintomatologia.

Por fim, faz-se necessário o desenvolvimento de novas pesquisas que visem a melhor abordagem adotada das dietas de eliminação e exclusão aos pacientes com EEo, observando os alimentos que influenciam na remissão dos eosinófilos, a fim 
de ter como objetivo final a melhoria quanto ao quadro clínico e estado nutricional dos pacientes. Desta forma contribuindo com profissionais da área, estudantes e pesquisadores.

\section{Referências}

Almeida, E. H. S. (2019). Efeito do treinamento muscular inspiratório na exposição ácida esofágica em pacientes com doença de refluxo gastroesofágico. Programa de Pós Graduação em Ciências Médicas. Fortaleza, Ceará, Brasil.

Arias, A., Pérez-Martínez, I., Tenías, J. M. \& Lucendo, A. J. (2016). Systematic review with meta-analysis: the incidence and prevalence of eosinophilic oesophagitis in children and adults in population-based studies. Alimentary Pharmacology and Therapeutics, 43, 3-15.

Barros, C. P.; Ferreira, C. T., \& Vieira, M. C. (2018). Esofagite Eosinofílica. Departamento Cientifico de Gastrenterologia, 3, 1-15.

Cezar, R. C., Nogueira, D. F., Valério, L. B., \& Passos, M. C. F. (2016). Caso 22. Revista Médica de Minas Gerais, 26, 1795e.

Cianferoni, A. (2016). Wheat allergy:diagnosis and management. Journal of Asthma and Allergy, 9,13-25.

Dellon, E. S., Liacouras, C.A., Molina-Infante, J., Furuta, G. T, Spergel, J. M., Zevit, N., Spechler, S. J, Attwood, S. E, Straumann, A., Aceves, S. S, Alexander, J. A, Atkins, D., Arva, N. C., Blanchard, C., Bonis, P. A., Book, W. M., Capocelli, K. E., Chehade, M., Cheng, E., Collins, M. H., \& Bredenoord, A. J. (2018). Critérios de diagnóstico de consenso internacional atualizados para esofagite eosinofílica: Procedimentos da Conferência AGREE. Gastroenterology, 155 (4), 1022-1033.e10.

Ferreira, C. T., Vieira, M. C., Furuta, G. T., Barros, F. C., \& Chehade, M. (2019). Eosinophilic esophagitis-Where are we today? J Pediatr (Rio J),95, 275 -81.

Fernandes, RA., Carraptosos, I., Faria, E., Pereira, C., \& Todo-Bom, A. (2018). Esofagite eosinofílica, alergia e défice de IgA-Cofatores ou cormobidades? Rev Port Imunoalergologia, 26(4), 285-290.

Gonsales, N., Yang, G. Y., Doerfler, B., Ritz, S., Ditto, A. M., \& Hirano, I. (2012). Elimination diet effectively treats eosinophilic esophagitis in adults; food reintroduction identifies causative factors. Gastroenterology, 142(7), 141-e15. doi.org/10.1053/j.gastro.2012.03.001

Groetch, M., Venter, C., Skypala, I., Vlieg-Boerstra, B., \& Grimshaw, K. (2017). Dietary therapy and nutrition management of eosinophilic esophagitis: a work group report of the American Academy of Allergy, Asthma, and Immunology. J Allergy Clin Immunol Pract, 5(2):312-24.

Higuera-Pulgar, I., Bretón-Lesmes, I., Carrascal- Fabián, M. L., Prieto- García, A., Menchén- Viso, L., Nogales-Ricón, O., Covadonga, I. H., Rodriguez, N. I., \& García- Peris, P. (2016). Ingesta dietética de pacientes adultos con esofagitis eosinofílica que siguen una dieta de exclusión de seis grupos de alimentos. Nutrición Hospitalaria, 33(4), 763-1012.

Hoversten, P., Kamboj, A. K., \& Katzka, D. A. (2018). Infections of the esophagus: an update on risk factors, diagnosis, and management. Dis Esophagus, 31(12), 1-9.

Kotchetkoff, E. C. A., Mendinça, R. B., Boaventura, R. M., Aranda, C. S., \& Sarni, R. O. S. (2020). Alergia alimentar ao leite de vaca e ovo mediado por IgE: sugestões de receitas termicamente testadas. Arq Asma Alerg Imunol, 4(4), 415-22.

Liacouras, C.A, Furuta, G. T., Hirano, I., Atkins, D., Attwood, S. E., Bonis, p. A. Burks, A. W., Chehade, M., Collins, m. H., dellon, E. S., Dohil, R., Falk, G. W., Gonsalves, N., Gupta, S. k., Katazka, D. A., Lucendo, A. J., Markowitz, J. E., Noel, R. J., Odze, R. D., Putnam, P. E., \& Aceves, S. S. (2011). Eosinophilic esophagitis: updated consensus recommendations for children and adults. The Journal of allergy and clinical immunology, $128(1), 3-22$.

Lucendo, A. J., Molina- Infante, J., Arias, Á., von Arnim, U., Bredenoord, A. J., Bussmann, C., Amil Dias, J., Bove, M., González- Cervera, J., Larsson, H., Miehlke, S., Papadopoulou, A., Rodríguez-Sánchez, J., Ravelli, A., Ronkainen, J., Santander, C., Schoepfer, A. M., Storr, M. A., Terreehorst, I., \& MolinaInfante, J., Fernando- Lamana, L., Ripoll, C., Hernandez- Alonso, M., Mateos, J. M., Fernandez-Bermejo, M., Dueñas, C., Fernandez-Gonzalez, N., Quintana, E. M., \& Gonzalez-Nuñez, M. A. (2011). Esophageal eosinophilic infiltration responds to proton pumb inhibition in most adults. Clinical gastroenterology and hepatology: the official clinical practice journal of the American Gastroenterological Associaciation, 9 (2), $110-117$.

Mendonça, L. P., \& Pinto, F. M. Esofagite eosinofílica na pediatria: um conceito e evolução. (2020). Braz. J. Hea. Rev. Curitiba, 3(1), 375-381,

Lucendo, A. J., Jesús, A. A., González-Cervera; J. L., Danila, G., Teresa, A., Jiménez-Contreras, Sonia González-Castillo, S., Rodríguez-Domíngez, B., Livia, C., \& Rezende, J. M. (2013). Empiric 6-food elimination diet induced and maintained prolonged remission in patients with adult eosinophilic esophagitis: A prospective study on the food cause of the disease. Journal of Allergy and Clinical Immunology, 131(3), 797-804.

Molina-Infante, J., Martin-Noguerol, E., \& Alvarado-Arenas M, et al. (2012). A dieta de eliminação seletiva com base em testes cutâneos tem um efeito subótimoficácia para esofagite eosinofílica em adultos. J Allergy Clin Immunol, 130 (5),1200-2.

Molina-infante, J.; Martíns-Noguerol, E., Alvarado-Arenas, M., Porcel-Carreño, S. L., Jimenez-Timon, S., \& Hernandez-Arbeiza, F. J. (2017). Dieta de eliminação empírica intensificada para esofagite eosinofílica pediátrica e adulta: O estudo 2-4-6. Alergia Alimentar e Doença Gastrointestinal,141(4), 13651372

Monteiro de Oliveira Cruz, R., Cruz, P. M. O., Machado, B. R. C., Lucena, T. C., D’Avila, S. P., \& Almeida, T. T. G. (2019). Visão histórica e fisiológica da interação do leite e do trigo com a microbiota intestinal humana. Revista Eletrônica Acervo Saúde, (27), e1026.

Pereira, A. S., Shitsuksa, D. M., Parreira, F. J., \& Shitsuka, R. (2018). Metodologia da pesquisa científica. UFSM.

Pesek, R. D., \& Gupta, S. K. (2018). Emerging drugs for eosinophilic esophagitis. Expert Opin Emerg Drugs, 23(2), $173-183$. 
Research, Society and Development, v. 10, n. 13, e588101321552, 2021 (CC BY 4.0) | ISSN 2525-3409 | DOI: http://dx.doi.org/10.33448/rsd-v10i13.21552

Pereira, A. H., Oliveira, A. K.S.; Salgado, F. S., \& Nunes, Carlos P. (2019). Esofagite Eosinofílica: Entidade Clínica Emergente ou Subdiagnosticada? Revista Caderno de Medicina, 2(1).

Reed, C. C., Fan, C., Koutlas, N. T., Shaheen, N. J., \& Dellon, E. S. (2017). Dietas de eliminação de alimentos são eficazes para o tratamento a longo prazo de adultos com esofagite eosinofílica. Alimentary Pharmacology and Therapeutics, 46 (9), 836-844.

Rawla, P., Sunkara, T., Thandra, K. C., \& Gaduputi, V. (2018). Effcacy and Safety of Budesonide in the Treatment of Eosinophilic Esophagitis: Updated Systematic Review and Meta-Analysis of Randomized and Non-Randomized Studies. Drugs in R\&D, (2):1-11.

Rienzo, T., D'angelo, G., D'aversa, F., Campanale, M., Cesario, V., Montalto, M., Gasbarrini, A., \& Ojetti, V. (2013). Lactose intolerance: from diagnosis to correct management. European Review for Medical and Pharmacological Sciences, 17(2), 18-25.

Rodriguez-Alarcon, C. A., Beristain- Ruiz, D. M., Rivera- Barreno, R., Díaz, G., Usón-Casaís, J. M., García-Herrera, R., \& Pérez-Merino, E. M. (2015). Gastroesophageal refluix in anesthetized dogs: a review. Revista Colombiana de Ciencias Pecuarias, 28(2), $144-155$.

Simon, D., Straumann, A. Wenk, A., Spichtin, H., Dimon, H. U., \& Braathen, L. R. (2006). Eosinophilic esophagitis in adults- no clinical relevance of wheat and rye sensitizations. Allergy, 61(2), 1480-3.

Straumann, A. (2017). Eosinophilic esophagitis: evidence-based stratements and recomendations for diagnosis and management in children and adults. United European Gastroenterol J, Apr; 5(3):335-358.

Teixeira, T. L., Linden, M. A., Lomazi, E. A., Saron, M. G. L., Riccetto, A. L., \& Bellomo- Brandão, M. A. (2020). Estudo caso-controle sobre o estado da vitamina D em crianças e adolescentes com esofagite eosinofílica. Arq. Gastroenterol, 57(4), 409-415.

Tovoli, F., Masi, C., Guidetti, E., Negrini, G., Paterini, P., \& Bolondi, L. (2015). Clinical and diagnostic aspects of gluten related disorders. World Journal of Clinical Cases, 3(3): 275-84.

Veiga, F. M. S., Castro, A. P. B. M., Santos, C. J. N., Dorna, M. B., \& Pastorino, A. C. (2017). Esofagite eosinofílica: um conceito em evolução? Arq Asma Alerg Imunol, 1(4):363-372. 